\title{
Research on 3D Model of Coal-bed Geologic Body
}

\author{
Feng Yongyu ${ }^{1,2, a}$, Mao $\mathrm{Xi}^{3, \mathrm{~b}}$ \\ ${ }^{1}$ China University of Mining and Technology (Beijing), Beijing, China \\ ${ }^{2}$ Shandong provincial land and Resources Information Center, Jinan, China \\ ${ }^{3}$ Chinese Academy of Surveying and mapping, Beijing, China \\ a fengyongyu@126.com, ${ }^{b}$ maoxi@casm.ac.cn
}

Keywords: Coal-bed Geologic Body, 3D, Data Structure, TIN

Abstract. The characteristics of the coal bed geological body and the commonly used 3D data model, the data model and the data model of the coal seam are studied. Based on this, a 3D data model is proposed, which is based on multi-layer TIN. The corresponding data structure is designed, and the algorithm of multi-layer TIN is given.

\section{Introduction}

Three dimensional data model is the concept and method of 3D spatial data organization; it reflects the spatial entities and their mutual relations in the real world. It is the research and development basis of 3DGM, 3DGIS and other fields [1,2], and is also a hot and difficult research in these areas. In 3D applications, the 3D data model can be divided into two categories: the surface-based data model representation and voxel-based representation model [3,4].

For coal mine application, it is hard to say which data model is the best because of the complexity of 3D space object. Because each data model has its own advantages and disadvantages and the scope of application. Based on this reason, this paper design a 3D model of coal-bed geologic body based on multi-layer TIN and propose the coal mine geological body generation algorithm.

\section{Data model of 3D geological body in coal mine}

From the introduction and review of the present situation of 3D data model, the model based on the data model and the data model have their own advantages and disadvantages, so it is a good choice to express complex spatial objects based on the hybrid structure. At present, the research on 3D data model based on hybrid structure has achieved some results, but it still cannot meet the practical application needs. The main problem is that these studies are mostly dedicated to provide a general data model for the 3DGIS, while few people conduct a data model of a professional field, which makes it difficult to implement the model, and cannot be generalized and applied. In order to avoid similar situation, this paper is devoted to the study of coal mine 3D geological objects, combined with the advantages of surface data model and data model based on data model, using the mixed model of multi-layer TIN and tetrahedral, which is used to express the simple, regular, three-dimensional geological body with a tetrahedral structure to express some complex and non-layered geological bodies. There are two reasons for using tetrahedral model as a complex geological structure, the first one is the complex geological structure does not have a layered structure and is not suitable to use the surface description method. The method of dividing the complex geological structure by using the method of dividing the body into a complex geological relationship can be expressed in the same way that the data quantity of the body can be reduced greatly. The second one is that multi-layer TIN can be easily transformed to tetrahedral partition model, so the generated geological body 3D model has the same logic, and meet the needs of some 3D attribute calculation and 3D space analysis.

Coal bed geological body is overall sequence distribution, and can be characterized by multiple surfaces, so each stratum can be characterized as surface regardless of fault, and can be simulated by the irregular grid. Stratum sometimes has the special structure such as fault, the fault data are mainly 
determined by the geophysical prospecting and other means. In the computer process, it usually depends on human computer interaction.

\section{Algorithm for generating 3D geological body of coal mine based on multi-layer TIN model}

Based on the research on the 3D data model and data structure of the coal stratum geological body, the algorithm of generating the 3D geological body of the coal mine by the multi-layer TIN model is proposed.

\section{(1) Determine the comprehensive stratigraphic sequence of the study area}

The first step of establishing the three-dimensional model of regional geological body is to determine the comprehensive stratigraphic sequence of the study area. Regional geological profile is a complex object, which reflects the geological structure of the exploration area, and is formed by the irregular structure expressing the stratigraphic structure. Therefore, the 3D modeling process of regional geological profile can be decomposed into the process of the formation, and finally form a complete three-dimensional model based on the strata expression. However, due to some constraints of the geological industry, and the establishment of a simple reasoning algorithm, in fact, the construction process is based on the synchronous analyses of three or more drilling data, and strata simultaneous deduction. The strata reasoning should comply with the regional stratigraphic distribution law. So it requires comprehensive hierarchical description data of the exploration area data as the basis. The integrated hierarchical description data reflects the sequence and distribution trend of a macro regional stratum. It provides the sequence relation of the abnormal stratum point out treatment in the process the stratum formation, and thus the uniqueness of the result is ensured. At the same time, we can find the attribute information of each layer in advance, according to the basic structure of the strata. The structure principle of the two dimensional section map can be expressed in Figure 1. Figure 1 is a comprehensive hierarchical description of the provisions of the stratigraphic sequence, so Figure 5 (a) expression is correct. In Figure 5 (b), It mistakes strata 1 to the ground 2, which violates the ranking rules of the strata in the comprehensive hierarchy description.

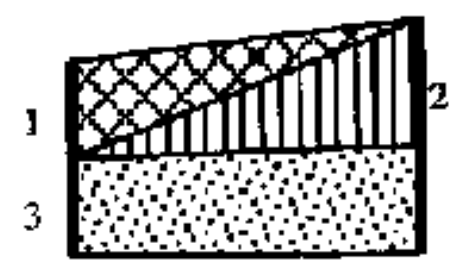

(a)

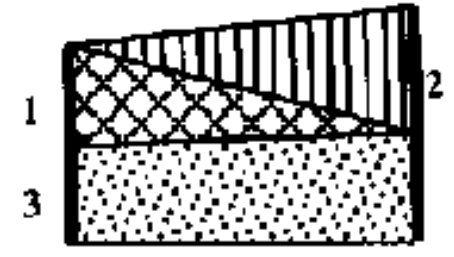

(b)

Fig. 1 The sketch map of comprehensive layer order

\section{(2) The triangulation of the drilling data and the encryption of the triangulation network}

It is necessary to set up a multi - layer TIN of the three - dimensional geologic body, but it is the same as the different strata.The establishment of the multi-layer TIN of three-dimensional geological body of need to carry out triangulation of the various strata. For stratigraphic samples are on the same location among the different strata, the triangulation of the various strata is the same without considering the case of the lack of drilling data. Due to the high cost of geological drilling, the actual amount of drilling data is limited, so the density of the triangle mesh is too thin. When the 3D geological body is expressed and the triangle network can not meet the needs, it is necessary to encrypt the triangle.

There are two kinds of encryption methods, the first one is a kind of computer automatic encryption, encryption for the incentre of each triangle, this method is simple and efficient, without manual intervention, and can meet all needs of any encryption, encryption and formation data also can be obtained by using different interpolation algorithms, but the disadvantage of this method that reliability of triangulation encryption is limited, the lack of professional knowledge control, and formation data greatly random; the second one is using the artificial intervention, manually select encryption sampling points, strata thickness and depth data of sampling points can be automatically 
calculated by the computer interpolation, and cann also according to the expert knowledge through expert manual interpretation of the sampling points in each stratum depth and the thickness data. This method is very professional, and the expert knowledge can be applied to 3D geological model, the disadvantage is that this method is inefficient, slow, and is not conducive to large areas of three-dimensional geological modeling.

We integrate these two methods mentioned above. It takes into account the shortcomings and strengths of the two methods. In the experiment, when the triangulation is carried out, the triangle mesh is used to insert the algorithm, and then it is optimized for the triangle net, which greatly reduces the computation.

\section{(3) Stratum triangulation}

After triangulation of the drilling data, we must carry out the analysis of the different strata according to the drilling triangulation. The different drilling strata data are not always the same as the research area, perhaps is lack of strata data, so each strata has to be processed.

The processing steps are as follows: the TIN points are compared with drilling data in the research of the regional strata,. If the TIN point is the same as drilling data, the TIN point is valid. If there is no point in the of TIN, the TIN point is valid. Then, the traversal all triangles of the triangulation.

According to whether the three vertices of the triangle in the triangle are valid points can be divided into 4 categories, as shown in Figure 2 (black points is valid, red point is not valid). For each triangle, if the three vertices of the triangle are all valid points, or are all invalid points (as a and $d$ in Figure 2), no treatment. In he other conditions, the midpoint of the valid points and invalid points (as $\mathrm{b}$ and $\mathrm{b}$ in Figure 2) is connected. After traversing all triangles of the triangulation, regional polygon of the lack of drilling is the midpoint connection region, as shown in figure 3 . Black point is the location of the drilling spot, the formation lack region is formed with orange point polygon. A new triangle net is formed by the edge of the area of the tip out of the area as the characteristic line. These orange points can also be determined by the addition of the formation of a pointed out line.

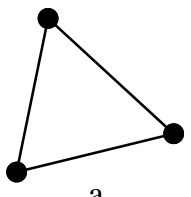

$\mathrm{a}$

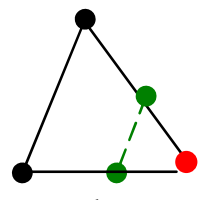

b

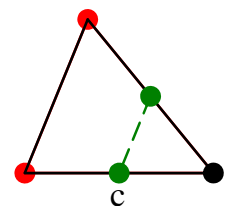

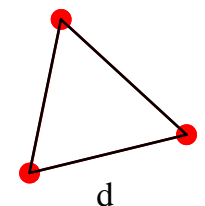

d

Fig. 2 The classification of triangles in triangle net

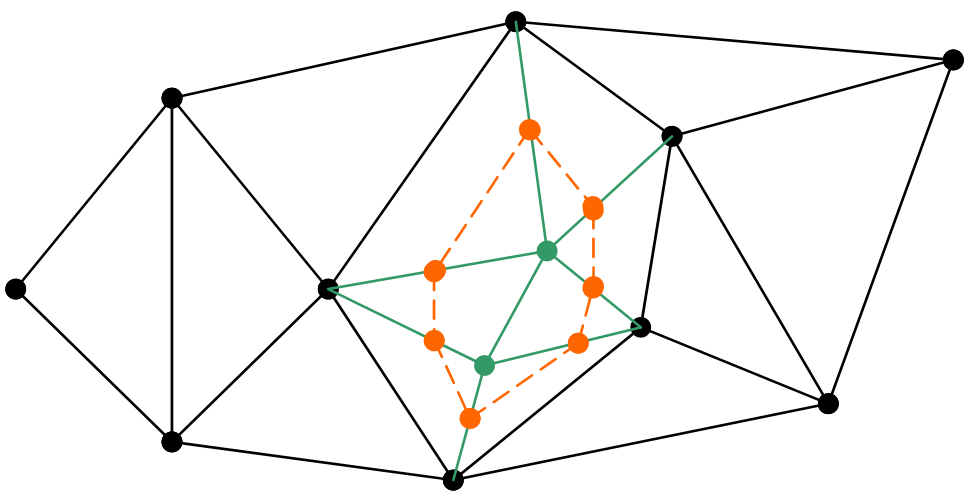

Fig. 3 The calculation sketch map of absent stratum

\section{(4) formation of the geological body}

According to the formation of multi layer TIN and the upper and lower floor information of of the strata, the geological body is simulated with the OpenGL graphics library. 


\section{Summary}

The key of constructing 3D data model and data structure of coal seam geological body. This paper first discusses the [10] on the basis of data model and data model. Based on the data model and data model, a new method is proposed, which is based on multi layer TIN to generate 3D data model.

The key to establish the coal mine virtual reality system is to construct the 3D data model and data structure which is suitable for the coal bed geological body. Firstly, the characteristics of the coal seam geological body and the commonly used 3D data model are discussed. Then, data model of 3D geological body in coal mine is designed.Finally, we propose the algorithm for generating 3D geological body of coal mine based on multi-layer TIN model. The results of this research will provide important theoretical basis and technical support for the construction of three-dimensional data model of coal mine virtual reality system and the development of coal mine virtual reality system.

\section{Acknowledgements}

This work was financially supported by Chinese Surveying and Mapping Public Industry Research Foundation (201412003) and Chinese Academy of Surveying and mapping Basic Research Foundation (7771417).

\section{References}

[1] Guo Peng, Wang Chao: 3D LOD Data Model of Underground Building and Structure and Its Application. Chinese Journal of Underground Space and Engineering, Vol.2 (2013), p.309

[2] Cheng Penggen: Research on There Dimensional Spatial Data Models and Algorithms in Geology and Mine (2005)

[3] Shi Yunfei:Hybrid Spatial Data Model for Three Dimensional cadaster. Journal of Remote Sensing, Vol.2(2013), p.327

[4] Wang Renhuai: Research on 3D Model of Mine Geological Object(2007) 